\title{
UN ÍNDICE DE POBREZA para Nuevo Casas Grandes, ChiHuahua
}

\author{
RICARDO LÓPEZ
}

HUGO DE LA TORRE

\section{RESUMEN}

E n este capítulo se construyó un índice de pobreza humana (IPH) para el municipio de Nuevo Casas Grandes, con el objetivo de medir sus niveles de pobreza, las causas de la misma así como su ubicación geográfica al interior de la localidad. El IPH es un índice de tipo multidimensional, puesto que incorpora cuatro aspectos interrelacionados para la medición de la pobreza: ingreso, educación, vivienda y salud. Adicionalmente, se analizaron los planes municipales de desarrollo (PMD) durante el periodo 2004-2016, que corresponden a cuatro administraciones locales, con la finalidad de caracterizar los ejes de política pública contenidos en los planes así como la visión de estos acerca de la pobreza y las acciones programáticas para su combate. Los resultados del IPH indican un nivel medio de pobreza, la cual, en términos absolutos, sufren alrededor de trece mil personas. Por su parte, en los PMD se observa la escasa atención por parte de estos hacia el problema de la pobreza, puesto que no ocupan un lugar central en la agenda de gobierno, en contraposición con otros aspectos como la seguridad pública, la infraestructura y el fomento económico. 
Palabras clave: Pobreza humana, Planes de desarrollo municipal, medición multidimensional, Política pública, Nuevo Casas Grandes.

\section{INTRODUCCIÓN}

Desde la conformación de los Estados-nación modernos, una de sus premisas centrales ha sido la búsqueda de bienestar para las personas. La definición de qué es el bienestar se puede considerar como un debate inacabado en parte a que el mismo está supeditado a un contexto de una sociedad específica y por lo tanto es compleja su generalización. En contraparte, existe un notable avance sobre qué no es el bienestar. Particularmente, se ha caracterizado ampliamente lo opuesto al bienestar, siendo el concepto más desarrollado el de pobreza. Ciertamente, la conceptualización de la pobreza no es un aspecto sencillo, ya que involucra la fijación y determinación de una serie de aspectos que son complejos de integrar. Sin embargo, a pesar de tal complejidad -o como producto de la misma- han surgido una serie de conceptualizaciones y metodologías orientadas a su medición que han sido bien aceptadas. Por ejemplo, el método de líneas de pobreza (MLP), el cual básicamente consiste en la fijación de un nivel mínimo de bienestar económico que se confronta con el ingreso promedio que percibe la persona o la familia para la determinación de si alcanza dicho nivel o no.

Autores como Paul Spicker, en su libro Las definiciones de pobreza: doce grupos de significados, señala que la conceptualización de la pobreza en las ciencias sociales al menos tiene doce acepciones. Una de estas es la relacionada con los aspectos materiales que poseen los individuos y las familias. Así, una persona es pobre o no en función de sus requerimientos materiales y que puede tener a su alcance o no. Lo interesante de la visión de Spicker et al. (1999), consiste en la acepción de que la pobreza se puede entender sencillamente como una privación de algo que la población necesita (alimentos, ropa, combustible, etcétera).

Claro está que al hablar de "privaciones" en el sentido estricto de la palabra, es preciso retroceder un poco y entender que lo que le antecede a una privación en sí debería corresponder a 
una necesidad que de momento o de manera permanente no ha sido satisfecha. Entonces, una necesidad claramente se relaciona con lo que percibimos y sentimos y que creemos se relaciona con nuestro bienestar, tanto objetivo como subjetivo. Con base en lo anterior, el índice de necesidades básicas insatisfechas (NBI), permite una mirada acerca de qué tipo de necesidades deberían ser cubiertas en una sociedad en determinado tiempo y contexto.

Sintetizando el razonamiento anterior, el NBI consiste en comparar la situación de cada hogar en materia de un grupo de necesidades específicas con una serie de normas que expresan, para cada una de ellas, el nivel mínimo debajo del cual se considera insatisfecha la necesidad. Los hogares con una o más NBI se consideran pobres, lo mismo que todos sus miembros (Boltvinik, 2000). Las necesidades consideradas tradicionalmente son: hacinamiento, vivienda inadecuada, abastecimiento inadecuado de agua, carencia o inconveniencia de servicios sanitarios para el desecho de excretas, inasistencia a escuelas primarias de los menores en edad escolar y un indicador indirecto de capacidad económica.

Como un intento de capturar los diversos aspectos del bienestar, en los últimos años se ha implementado a escala mundial una metodología orientada a conceptualizar y medir a la pobreza desde una visión integral (en paralelo al NBI, que también se puede considerar como un esfuerzo pionero en el ámbito multidimensional), conocida como multidimensional. Dicha metodología se caracteriza por conceptualizar al fenómeno como una expresión y consecuencia de una serie de aspectos que se circunscriben no solamente al ingreso (como lo hace el método de líneas de pobreza), sino a toda una serie de factores y aspectos interrelacionados bajo un mismo problema: la pobreza.

En el enfoque del bienestar, el objetivo primordial radica en identificar las dimensiones y condiciones que limitan la libertad de las personas para desarrollarse plenamente. Se supone que cada persona, a partir de sus circunstancias y preferencias, desarrolla un conjunto de capacidades que definen el abanico de opciones de vida que puede elegir. Si estas opciones no le permiten tener condiciones de vida aceptables dentro de una sociedad, se considera al individuo pobre (Coneval, 2009: 27). 
En cuanto a las dimensiones sociales, se plantea que todos los seres humanos por el solo hecho de existir tenemos derecho a acceder a un conjunto de valores, intereses y bienes que, por su urgencia e importancia, deben ser considerados como fundamentales y comunes a todos los seres humanos. De esta forma, se considera que toda persona debe contar con una serie de garantías indispensables para la dignidad humana que, al ser adoptadas dentro del marco jurídico nacional o al ser ratificadas mediante la firma de los instrumentos internacionales que las amparan, se convierten en obligaciones para el Estado, el cual deberá generar los mecanismos que permitan, progresivamente, el acceso pleno a los derechos humanos.

Dicho lo anterior, en México, al igual que en la mayor parte de los países a escala planetaria, el bienestar de sus habitantes y por ende su complemento, reducción o eliminación de la pobreza, ha motivado la implementación de diversas políticas de corte social y económico. Por ejemplo, desde el inicio de la década de los ochenta se pusieron en práctica políticas de asistencia social para garantizar el consumo, abasto y distribución de alimentos, al mismo tiempo que se promovía una reestructuración agrícola y una mayor actividad de los flujos comerciales de productos alimenticios con el exterior en aras de permitirle al país la importación de alimentos "baratos" que favorecieran el acceso a los mismos a la población de más bajos recursos. Desde entonces adquirió particular relevancia, en los programas de política social del Estado mexicano, el combate a la pobreza extrema y/o pobreza alimentaria, en gran parte debido a la influencia que en ese momento adquirieron las discusiones relativas al hambre y la pobreza, así como los derechos y las garantías de acceso y calidad de los alimentos.

Sin embargo, cabe decir que la evolución de la pobreza en México no ha sido la óptima, ya que entre 1963 y 1977, la pobreza extrema disminuyó en casi $5 \%$, al pasar de 26.7 millones a 21.5 $\%$, situación que se atribuye al auge petrolero y a las tasas moderadas de inflación. Sin embargo, para 1988 la pobreza extrema aumentó de nuevo a 23.2 millones y se incrementó a 35.1 millo- 
nes en 1996 como resultado de la crisis económica de $1995 .{ }^{1}$ Con el inicio del nuevo milenio, la pobreza extrema se redujo a 11.6 millones, los pobres no extremos a alrededor de 10 millones y el total de pobres a 21.3 millones. Para 2010 la pobreza extrema descendió de nuevo en 2.3 millones, comparado con la cifra previa; sin embargo, los pobres no extremos y la pobreza absoluta se incrementaron en 7.7 y 5.3 millones, respectivamente.

En dicho contexto, la pobreza en México sigue representando uno de los problemas más agudos que condicionan las posibilidades de desarrollo de la población. No obstante, creemos pertinente particularizar el análisis hacia la unidad administrativa más cercana a los ciudadanos, la cual a nivel constitucional corresponde al municipio. En relación a la incidencia de la pobreza a nivel municipal, de los 2442 municipios existentes en México, 1063 (44 \% del total) forman parte del padrón del Programa Alimentario (programa que a partir de 2016 se fusiona con Prospera) y Abasto Rural (PAR, operado por la Secretaría de Desarrollo Social, Federal), y casi todos ellos son cubiertos por el Programa Oportunidades (renombrado Prospera) y por la Cruzada contra el Hambre.

En este sentido, alrededor de la mitad de los municipios en el país enfrenta algún grado de pobreza, aunque difieren en grado de intensidad. Por ejemplo, es bien conocido que en México las entidades federativas del sur y sureste tienden a concentrar en mayor medida la pobreza extrema, también caracterizada como alimentaria. En tanto, en las entidades situadas en el centro y norte, los niveles de pobreza suelen ser menores, sobre todo la extrema y alimentaria. Lo anterior ha motivado la afirmación de que presenciamos la emergencia de dos países opuestos, tomando como referencia sus niveles de desarrollo. Sin embargo, desde nuestra óptica, consideramos dicha afirmación como inexacta y descontextualizada, debido a que el norte y el centro del país también muestran una gran heterogeneidad que impide su generalización en términos de desarrollo.

1 Durante dicho año el PIB decreció alrededor del $6 \%$ respecto al año previo; por mucho, una de las caídas económicas más relevantes del país en las últimas tres décadas. 
Precisamente, el objetivo central del capítulo consiste en analizar el grado de pobreza humana en el municipio de Nuevo Casas Grandes, ${ }^{2}$ perteneciente al estado de Chihuahua. A pesar de la abundante disponibilidad de datos oficiales (gracias al Coneval), decidimos construir un índice de pobreza humana (IPH) tomando como referencia una metodología utilizada por el Programa de las Naciones Unidas para el Desarrollo (PNUD). Dicha metodología concibe a la pobreza como un efecto que resulta de la interrelación de cuatro elementos: ingreso, salud, educación y vivienda. Si bien el índice bien se puede nombrar como multidimensional, gracias a su construcción a nivel de área geoestadística básica $(\mathrm{AGEB})^{3}$ permite ubicar espacialmente la intensidad de la pobreza así como la variable que tiene mayor influencia dentro de la misma. Con ello, desde nuestra perspectiva, se favorece la aplicación de políticas públicas diseñadas a la medida para combatir el problema desde lo local.

Adicionalmente, analizamos cuáles han sido las estrategias y acciones de política pública propuestas por el municipio en aras de mitigar o combatir el fenómeno de la pobreza a escala local. La fuente primaria de información para cumplir lo anterior le corresponde a los Planes de Desarrollo Municipal de Nuevo Casas Grandes, implementados durante el periodo 2004-2016, es decir, cuatro trienios de administración municipal.

La estructura del documento es la siguiente: en la primera sección se muestra una breve caracterización del municipio en el país, con la premisa de posicionar el grado de avance de los mismos dentro de los órdenes gubernamentales. La segunda sección se orienta a caracterizar los planes de desarrollo municipal

2 Este capítulo se desprende de un proyecto más amplio titulado "Construcción de un índice de Pobreza Humana en dos municipios ferrocarrileros del noroeste de México, 2000-2010". Proyecto de incorporación de nuevos profesores de tiempo completo, (Prodep), Clave: DSA/103.5/14/7303, a cargo del doctor Ricardo López Salazar. Básicamente, el citado proyecto compara la evolución de la pobreza humana en Nuevo Casas Grandes y Empalme, Sonora, debido a que ambos municipios nacieron con el ferrocarril, por lo que la intención primaria consistió en analizar cómo se modificó el IPH al concluir la actividad ferroviaria en 1999 (totalmente para Nuevo Casas Grandes y de manera parcial en Empalme, puesto que se mantiene el ferrocarril de carga).

3 Más adelante se explicará con detalle que es un AGEB. 
del municipio de estudio, así como las políticas emanadas de los mismos, relacionadas con el combate a la pobreza. La tercera sección aborda con mayor detalle qué representa el IPH y la metodología utilizada para su construcción; asimismo, se presentan y analizan los resultados obtenidos al aplicar la metodología del IPH para Nuevo Casas Grandes. Finalmente, exponemos algunas conclusiones sobre el tema tratado.

\section{BREVE CARACTERIZACIÓN DEL MUNICIPIO EN MÉXICO}

A medida que avanza la conceptualización de la ciencia regional, se reconoce en mayor grado a los gobiernos locales como unidades indispensables en la planeación de políticas dirigidas a solucionar problemáticas locales. La premisa de aplicar políticas locales a problemas locales se sustenta en que las localidades y las regiones son ámbitos cotidianos cercanos a todo aquello que las personas ven como más próximo: educación, salud y vivienda, entre otros (Boisier, 1998).

En el caso de México, el municipio ha sido estudiado desde diversos enfoques, cada cual atendiendo la especificidad concreta de la problemática abordada. Dentro de la variada agenda de estudios municipales resaltan al menos tres o cuatro líneas de investigación recurrentes. Los que se inclinan a estudiar la intervención del municipio en la conformación del sistema urbano nacional, como Garza (2006) y Ziccardi (2008), quienes encuentran que los municipios, y en especial sus gobiernos, se enfrentan a una nueva etapa en su desarrollo caracterizada por la constante competencia entre ciudades por atraer inversión, la creciente pobreza y desigualdad provocada en gran medida por la precariedad e informalidad del empleo. Así, el reto de los gobiernos locales se centra en impulsar y consolidar políticas dirigidas a materializar un desarrollo integral e incluyente de todos los actores sociales, políticos y civiles.

Otra corriente se enfoca en estudiar la construcción de la agenda municipal y como esta determina en buena medida la trayectoria del municipio hacia el cumplimiento de sus responsabilidades y obligaciones como nivel de gobierno. Guillén (2005) sitúa a la 
agenda municipal como el mecanismo que introduce los temas que posteriormente se convierten en las acciones de política llevados a cabo en las administraciones locales. De esta forma, incluso en el ámbito municipal, los planes y programas de política implementados envuelven procesos de maduración. Al respecto, Arellano (2000) señala que la agenda municipal es un instrumento importante para proyectar y ordenar las prioridades que el proceso de modernización municipal y local exige. En términos generales parece observarse una tendencia hacia la formalización e institucionalización de dicha agenda (Arellano, 2000: 24).

Tal vez la temática más explorada en los estudios municipales sea el papel del municipio en el desarrollo del país. Este punto, que de primera instancia suena muy general, involucra varios subtemas, que bien se pueden clasificar en: el papel del municipio en la democratización de México; el municipio como planeador del desarrollo local; los desafíos que enfrenta el municipio con el proceso de descentralización. Por razones de espacio e interés para nuestro tema, solo abordaremos el segundo y el tercer subtemas.

Los municipios como planeadores, sin duda, se enmarcan en la concepción del espacio local como una unidad activa en la promoción del desarrollo. En otras palabras, se parte del reconocimiento de que la planeación local y regional ofrece un enfoque alternativo para la promoción del desarrollo regional desde "adentro" donde las decisiones tendrán efecto (Gore, 1984). Así, las políticas de desarrollo local persiguen el objetivo de aliviar las disparidades, fomentar la equidad espacial e involucrar a la gente en los procesos de desarrollo, por lo que el criterio para evaluar el éxito de una política local y regional es la reducción de las disparidades en los niveles de bienestar de la población (ídem).

El estudio de Cabrero (2005), habla de acciones públicas de diferente intensidad, las cuales están determinadas por diferentes arreglos institucionales y se encuentran en una etapa de construcción permanentemente, independientemente de la cualidad del espacio social determinado. Los beneficios que puede traer la acción pública eficiente son la generación de confianza y la convergencia de los esfuerzos individuales hacia los colectivos, que deriven en la maduración de estrategias claras de desarrollo local. 
Por su parte, Merino (2006) sitúa al municipio como una de las piezas clave para el desarrollo del país desde el surgimiento del movimiento de independencia (Merino, 2005). Sin embargo, también reconoce la pérdida de espacios de poder y su subordinación hacia los niveles de gobierno estatal y federal. Dicha subordinación provocó que los municipios, ni con mucho, intervinieran en los procesos de planeación de políticas en la etapa del modelo sustitutivo de importaciones.

Gutiérrez Vidal y Martínez Pellegrini (1994) categorizan al municipio como un espacio propicio para impulsar el desarrollo a partir de sus propias potencialidades endógenas, las cuales no pueden ser reproducibles en otros contextos debido a sus especificidades internas, que demandan un diseño original y único para cada municipio. Adicionalmente, señalan la carencia de recursos financieros en los municipios, factor que limita cumplir las nuevas funciones que le han sido transferidas por el gobierno central.

Mención aparte merecen los estudios que abordan el proceso de descentralización municipal. Este amplio espectro de estudios tomó un nuevo impulso con las reformas al artículo 115, efectuadas en 1983 y 1999, que reconocieron al municipio de la siguiente manera: el municipio es la institución político-jurídica integrada por una población que comparte identidades culturales, históricas y un idioma común, asentada en un territorio delimitado que se administra por autoridades constituidas en un Ayuntamiento electo por sufragio universal y directo, para su progreso y desarrollo.

En este sentido, se reconoce al municipio como la entidad jurídica más cercana a los ciudadanos y por ende la que mejor puede resolver los problemas de los mismos, así como la base para el desarrollo de los estados y la nación. Sin embargo, los resultados arrojados por estudios como el de Merino (2005) reporta que el municipio permanece como la unidad de gobierno más endeble del sistema federal mexicano debido a los problemas de financiamiento, lo limitado de sus recursos humanos y la ausencia de técnicas eficientes de gestión pública.

En cuanto al financiamiento, el municipio mantiene una alta dependencia de los recursos que se le transfieren, como participaciones federales, aunque con una diferenciación en cuan- 
to al grado de dependencia entre los municipios con muy baja marginación (571 predominantemente urbanos) y muy alta marginación (1867 predominantemente rurales). En el primer caso, representan $58.12 \%$ de los ingresos totales, mientras que para el segundo, representan el $85.1 \%$. Los ingresos propios generados, para los municipios agrupados en muy baja marginación, ascienden a $26 \%$ del total, y apenas a $9.5 \%$ para los agrupados como de muy alta marginación (Merino, 2006).

Los recursos humanos, dentro de las administraciones municipales, no son abundantes. Para muestra lo siguiente: tres de los principales niveles jerárquicos, como el presidente municipal, los secretarios y tesoreros, solo habían cursado estudios a nivel de licenciatura con cerca del 45, 47 y $29 \%$, respectivamente. Además, la carestía de experiencia previa en cargos públicos de los mandos altos, con un valor de $66 \%$. Adicionalmente, las tasas de rotación de los servidores públicos son altísimas, ya que alcanzan el $95 \%$ cada tres años.

El resultado se configura en la no-generación de trayectorias de aprendizaje en la administración pública municipal, factor clave en la ausencia de técnicas eficientes de gestión pública. Un factor que favorece las ausencias en las técnicas de gestión son las diferencias en los periodos en el poder entre los gobiernos municipales, de solamente tres años, contra seis de los gobiernos estatales y federal. Por lo tanto, las trayectorias de aprendizaje son heterogéneas a nivel intergubernamental, y los gobiernos municipales son el actor más endeble (ídem).

\section{ASPECTOS GENERALES DE LOS PLANES DE DESARROLLO MUNICIPAL}

\section{Estructura}

La estructura de los planes de desarrollo analizados muestra amplias coincidencias entre sí. En primer lugar, estos inician con una parte descriptiva del marco legal donde se sustentan el derecho 
y las obligaciones de la planeación municipal. ${ }^{4}$ En este sentido, los planes abordan con mayor o menor extensión (según sea el caso) algunos de los aspectos contenidos en la ley de planeación del estado. En segundo lugar, se encuentra el mensaje del alcalde donde se detalla a grandes rasgos el contenido del plan y la forma como este intenta responder a las demandas de la ciudadanía.

La descripción del marco legal, así como el mensaje emitido por el alcalde, se configuran en la parte introductoria de los planes. Pasado esto, se comienza con un diagnóstico o descripción de manera breve y general de las principales actividades económicas desarrolladas en los municipios. A manera de marco contextual, también se incluyen aspectos geográficos y demográficos que dan cuenta del crecimiento poblacional y su consecuente reto para la proveeduría de servicios públicos. Esta fase bien puede ser considerada como el estado del municipio al momento de la asunción de las nuevas autoridades gubernamentales.

\section{Ejes de política y diagnóstico de la pobreza}

Con base en el diagnóstico, se derivan los principales problemas que aquejan a la población, los cuales en un primer momento se configuran en ejes u objetivos a perseguir en el periodo de gestión de la autoridad en turno. Dichos ejes u objetivos se acompañan de líneas de política donde se explicita la forma en que la autoridad gubernamental resolverá o al menos intentará darle solución al problema identificado. Por su parte, las líneas de acción se acompañan de estrategias desglosadas con la finalidad de atacar el problema para el cual haya sido desarrollada.

De acuerdo a los PDM analizados, se vislumbra que la "noción del desarrollo", sea social, económico, humano o rural ocupa un lugar central en los mismos. Solamente el PDM (2010-2013) sitúa el combate a la pobreza como un eje de política y asimismo se diagnostica la necesidad de implementar acciones encaminadas

4 En términos generales, los planes estudiados describen algunos de los artículos contenidos en leyes como la constitución, la ley de planeación y la ley de gobierno y administración pública del estado. Sin embargo, cada uno de los planes explora en mayor o menor grado el marco legal en el cual se sustenta la planeación municipal. 
a mejorar la calidad de vida de la población. Sin embargo, ninguno de los planes otorga información estadística que permita dimensionar la magnitud del problema, por lo que se quedan en un intento meramente exploratorio e ilustrativo del fenómeno.

Cuadro 1. Ejes de política de los Planes de Desarrollo Municipal (PDM) y diagnóstico de la pobreza en Nuevo Casas Grandes, 2004-2016

\begin{tabular}{|c|c|c|c|c|}
\hline $\begin{array}{l}\text { Nuevo } \\
\text { Casas } \\
\text { Grandes }\end{array}$ & $\begin{array}{l}\text { PDM } \\
\text { (2004-2007) }\end{array}$ & $\begin{array}{l}\text { PDM } \\
(2007-2010)\end{array}$ & $\begin{array}{l}\text { PDM } \\
(2010-2013)\end{array}$ & $\begin{array}{l}\text { PDM } \\
(2013-2016)\end{array}$ \\
\hline $\begin{array}{l}\text { Ejes de } \\
\text { política }\end{array}$ & $\begin{array}{l}\text { 1. Salud } \\
\text { 2. Servicios } \\
\text { públicos } \\
\text { 3. Actividad } \\
\text { económica } \\
\text { 4. Gobierno } \\
\text { 5. Estructura y } \\
\text { organización de } \\
\text { la administra- } \\
\text { ción municipal } \\
\text { 6. Seguridad } \\
\text { pública } \\
\text { 7. Fomento } \\
\text { económico } \\
\text { 8. Obras públi- } \\
\text { cas } \\
\text { 9. Desarrollo } \\
\text { social }\end{array}$ & $\begin{array}{l}\text { 1. Desarrollo } \\
\text { humano } \\
\text { 2. Desarrollo } \\
\text { social } \\
\text { 3. Desarrollo } \\
\text { económico } \\
\text { 4. Desarrollo } \\
\text { regional } \\
\text { 5. Justicia y } \\
\text { seguridad } \\
\text { 6. Cultura y ca- } \\
\text { lidad educativa }\end{array}$ & $\begin{array}{l}\text { 1. Desarrollo } \\
\text { social } \\
\text { 2. Asistencia } \\
\text { social del DIF } \\
\text { municipal } \\
\text { 3. Combate a la } \\
\text { pobreza } \\
\text { 4. Seguridad } \\
\text { pública } \\
\text { 5. Protección } \\
\text { civil } \\
\text { 6. Derechos } \\
\text { humanos } \\
\text { 7. Organizacio- } \\
\text { nes civiles } \\
\text { 8. Salud } \\
\text { 9. Atención a } \\
\text { grupos vulne- } \\
\text { rables }\end{array}$ & $\begin{array}{l}\text { 1. Salud } \\
\text { 2. Desarrollo } \\
\text { social } \\
\text { 3. Desarrollo } \\
\text { rural } \\
\text { 4. Obras públi- } \\
\text { cas } \\
\text { 5. Fomento } \\
\text { económico } \\
\text { 6. Comercio y } \\
\text { servicios } \\
\text { 7. Desarrollo } \\
\text { urbano } \\
\text { 8. Educación } \\
\text { 9. Seguridad } \\
\text { pública }\end{array}$ \\
\hline $\begin{array}{l}\text { Diagnós- } \\
\text { tico de la } \\
\text { pobreza }\end{array}$ & $\begin{array}{l}\text { No se encuen- } \\
\text { tra un diagnós- } \\
\text { tico específico, } \\
\text { pero destaca la } \\
\text { necesidad de } \\
\text { mejorar en los } \\
\text { aspectos de la } \\
\text { salud, sector } \\
\text { económico pri- } \\
\text { mario y seguri- } \\
\text { dad pública. }\end{array}$ & $\begin{array}{l}\text { El plan especi- } \\
\text { fica que el nivel } \\
\text { de vida en el } \\
\text { municipio ha } \\
\text { mejorado no- } \\
\text { tablemente, un } \\
\text { alto porcentaje } \\
\text { de la población } \\
\text { de las zonas } \\
\text { rural y urbana } \\
\text { están siendo } \\
\text { atendidos en } \\
\text { forma integral. }\end{array}$ & $\begin{array}{l}\text { El plan señala } \\
\text { que en el mu- } \\
\text { nicipio es nece- } \\
\text { saria la pronta } \\
\text { búsqueda de } \\
\text { soluciones a las } \\
\text { demandas so- } \\
\text { ciales que per- } \\
\text { mitan mejorar } \\
\text { la calidad de } \\
\text { vida de quienes } \\
\text { más lo necesi- } \\
\text { tan. }\end{array}$ & $\begin{array}{l}\text { No se encuen- } \\
\text { tra un diagnós- } \\
\text { tico específico, } \\
\text { pero se enfatiza } \\
\text { la necesidad de } \\
\text { proporcionar a } \\
\text { la comunidad } \\
\text { un ambiente } \\
\text { de oportu- } \\
\text { nidad para } \\
\text { desarrollarse } \\
\text { promoviendo el } \\
\text { bienestar social } \\
\text { e integral entre } \\
\text { los grupos más } \\
\text { vulnerables. }\end{array}$ \\
\hline
\end{tabular}

Fuente: Martínez, 2015 


\section{Objetivos y estrategias de políticas propuestas para el combate a la pobreza}

Los objetivos plasmados en relación con el combate a la pobreza son coincidentes en que, a grandes rasgos, todos ellos buscan elevar o mejorar el nivel y calidad de vida de la población, sin embargo, exhiben diferentes estrategias para logar su cometido. Así, dos de ellos (2004-2007, 2010-2013) se orientan hacia el rubro asistencial, como gestionar recursos para las despensas y para el programa alimentario, mientras los dos restantes (2007-2010 y 2013-2016) esbozan políticas diferenciadas, orientadas a impulsar el autoempleo y la recuperación de los espacios urbanos en aras de fortalecer el desarrollo comunitario (véase cuadro 2). 
Cuadro 2: Políticas de combate a la pobreza propuestas en los Planes Municipales de Desarrollo (PDM) en Nuevo Casas Grandes, 2004-2016

\begin{tabular}{|c|c|c|c|c|}
\hline $\begin{array}{l}\text { Nuevo } \\
\text { Casas } \\
\text { Grandes }\end{array}$ & $\begin{array}{l}\text { PDM (2004- } \\
2007)\end{array}$ & $\begin{array}{l}\text { PDM (2007- } \\
2010)\end{array}$ & $\begin{array}{l}\text { PDM (2010- } \\
2013)\end{array}$ & $\begin{array}{l}\text { PDM (2013- } \\
2016)\end{array}$ \\
\hline Objetivos & $\begin{array}{l}\text { Apoyar a la } \\
\text { población de } \\
\text { escasos re- } \\
\text { cursos en el } \\
\text { abastecimiento } \\
\text { de productos } \\
\text { de consumo } \\
\text { básico. }\end{array}$ & $\begin{array}{c}\text { Promover, im- } \\
\text { pulsar y gestio- } \\
\text { nar acciones, } \\
\text { obras o servi- } \\
\text { cios tendientes } \\
\text { a mejorar las } \\
\text { condiciones de } \\
\text { vida en materia } \\
\text { de vivienda y } \\
\text { servicios, aten- } \\
\text { diendo priorita- } \\
\text { riamente a los } \\
\text { grupos vulne- } \\
\text { rables. }\end{array}$ & $\begin{array}{c}\text { Desarrollar } \\
\text { acciones } \\
\text { tendientes al } \\
\text { fortalecimiento } \\
\text { de los diversos } \\
\text { programas e } \\
\text { instituciones } \\
\text { dedicados a la } \\
\text { asistencia en el } \\
\text { municipio. }\end{array}$ & $\begin{array}{c}\text { Brindar aten- } \\
\text { ción prioritaria } \\
\text { a los sectores } \\
\text { en pobreza } \\
\text { extrema y zo- } \\
\text { nas rurales del } \\
\text { municipio. }\end{array}$ \\
\hline $\begin{array}{l}\text { Estrate- } \\
\text { gias de } \\
\text { combate } \\
\text { a la po- } \\
\text { breza pro- } \\
\text { puestas }\end{array}$ & $\begin{array}{c}\text { Gestionar el } \\
\text { subsidio de des- } \\
\text { pensa básica } \\
\text { en periodos de } \\
\text { emergencia y } \\
\text { vulnerabilidad } \\
\text { social. }\end{array}$ & $\begin{array}{l}\text { Promover el } \\
\text { financiamiento } \\
\text { de proyectos } \\
\text { productivos en } \\
\text { colonias popu- } \\
\text { lares, que brin- } \\
\text { den ingresos } \\
\text { complementa- } \\
\text { rios, prioritaria- } \\
\text { mente a perso- } \\
\text { nas en pobreza } \\
\text { extrema. }\end{array}$ & $\begin{array}{l}\text { Fortalecer } \\
\text { el programa } \\
\text { alimentario, } \\
\text { beneficiando } \\
\text { a familias, } \\
\text { adultos mayo- } \\
\text { res, mujeres } \\
\text { embarazadas y } \\
\text { discapacitados, } \\
\text { con desnutri- } \\
\text { ción o riesgo de } \\
\text { desnutrición. }\end{array}$ & $\begin{array}{c}\text { Impulsar la } \\
\text { construcción, } \\
\text { remodelación } \\
\text { y reconstruc- } \\
\text { ción de centros } \\
\text { comunitarios } \\
\text { que permitan } \\
\text { implementar } \\
\text { actividades que } \\
\text { promuevan el } \\
\text { desarrollo y la } \\
\text { convivencia de } \\
\text { la población. }\end{array}$ \\
\hline $\begin{array}{l}\text { Depen- } \\
\text { dencias } \\
\text { encarga- } \\
\text { das de } \\
\text { aplicar las } \\
\text { estrate- } \\
\text { gias }\end{array}$ & $\begin{array}{c}\text { Desarrollo So- } \\
\text { cial } \\
\text { Protección Civil } \\
\text { del Estado } \\
\text { Gobierno } \\
\text { Federal } \\
\text { Comités de } \\
\text { Vecinos } \\
\text { Asociaciones } \\
\text { Civiles }\end{array}$ & $\begin{array}{c}\text { Desarrollo } \\
\text { Social } \\
\text { Gobierno } \\
\text { Federal }\end{array}$ & DIF municipal & No se especifica \\
\hline
\end{tabular}

Fuente: Martínez, 2015 


\section{EL ÍNDICE DE POBREZA HUMANA}

La pobreza en general significa una reducción en las posibilidades de acceso a bienes materiales y de ingreso por parte de las personas para poder tener una mejor calidad de vida y, por tanto, se configura como un problema continuo, cíclico y rodeado de múltiples dimensiones. Puede implicar no solo la falta de los artículos necesarios para el bienestar material, sino la denegación de la oportunidad de vivir una vida tolerable (PNUD, 1997). Entonces la pobreza, al contrario de lo que estipula el desarrollo humano, representa un acotamiento o incluso la imposibilidad que sufren las personas para poder desenvolverse dentro de los parámetros considerados como "dignos" dentro de una sociedad específica.

Sin embargo, uno de los conflictos que envuelve la conceptualización de la pobreza (y su posterior medición), se atribuye a la dificultad para establecer parámetros o indicadores del desarrollo de la sociedad y su vinculación con los satisfactores que deben considerarse como mínimamente aceptados. ${ }^{5}$ Incluso, es común que el desarrollo de los estratos de la población más altos encubran la involución de los estratos de la población menos favorecida. Es por ello que la pobreza y el desarrollo son dos aspectos que aunque a priori parecería sencillo conjuntarlos, en la práctica aparecen como una de las uniones más complejas.

El contraste entre desarrollo humano y pobreza humana refleja dos maneras diferentes de evaluar el desarrollo. Una manera, la "perspectiva conglomerativa", ${ }^{6}$ se concentra en los adelantos de todos los grupos de cada comunidad, desde los ricos hasta los pobres. Esto contrasta con otro punto de vista, "la perspectiva de privación", en la que se juzga el desarrollo por la forma en que viven en cada comunidad los pobres y las personas privada de recursos. La falta de progreso en la reducción de las desventajas de las personas privadas de recursos no puede ser "borrada" por grandes adelantos -por grandes que sean- de la gente que vive

\footnotetext{
5 Véase Salama (2011).

6 Desde esta perspectiva se analizan los avances de la sociedad de manera conjunta. Sin embargo, no se contabilizan de esa manera, sino que se evalúan estratos por separado para evitar omisiones o sobreestimaciones.
} 
mejor (PNUD, 1997). Entonces, la propuesta del Índice de Pobreza Humana radica en la posibilidad de medir (mediante cuatro variables) las condiciones de vida de las personas. Dichas variables son: el ingreso, la educación, los servicios dentro de la vivienda y el acceso a la salud.

Por supuesto que el IPH no agota todas las posibilidades existentes para medir la pobreza, sin embargo, sí intenta al menos incorporar tres de los enfoques más utilizados para medir la pobreza en el ámbito académico. En adición, incorpora algunos de los elementos propuestos en el Índice de Desarrollo Humano (IDH), propuesto por Amartya Sen (1999) y el Programa de Naciones Unidas para el Desarrollo (PNUD). Dicho índice combina tres elementos para evaluar el progreso de los países en materia de desarrollo humano: el Producto Interno Bruto (PIB) por habitante, la salud y la educación; cada uno se incluye con la misma ponderación.

Por ejemplo, se incorpora el ingreso, el cual por sí mismo ha representado uno de los enfoques más utilizados para medir la pobreza. Asimismo, se incluyen la educación y la salud, los cuales son aspectos que se relacionan con las necesidades básicas, ya que van más allá del ingreso debido a que involucran la capacidad de los servicios prestados por el Estado o por las comunidades para que sus habitantes o pobladores no caigan en situación de pobreza. Por último, la inclusión de los servicios dentro de la vivienda se relaciona con las capacidades de las personas para satisfacer de manera adecuada uno de los aspectos mínimos que se necesitan para vivir dignamente.

\section{La metodología utilizada para la construcción del IPH}

La fuente de información central para la elaboración del índice provino del Censo General de Población y Vivienda elaborado por el INEGI en 2010. De manera específica, se desagregó la información de acuerdo a la forma más utilizada por INEGI que es el AGEB. Al estimar el IPH se consideró toda área geoestadística básica (AGEB) para el cálculo del indicador en función de su delimitación urbana. De acuerdo con INEGI, un AGEB comprende la circunscripción territorial que concierne a la subclasificación de áreas geoestadís- 
ticas municipales, y que en nuestro caso incluyen indicadores de población, fecundidad, vivienda, salud, educación y empleo.

Dentro de la información disponible de INEGI, tanto las áreas AGEB como las localidades comprenden una extensión en función del número de habitantes. Toda AGEB con un número reducido de habitantes carece de información, por ello se descartaron aquellas señaladas con un número de habitantes que no representara información de los indicadores de interés. De esta manera, con los AGEB que ofrecen disponibilidad de datos se procede a al cálculo y construcción del IPH, el cual se compone de cuatro subíndices que se orientan a medir el ingreso, la educación, los servicios de la vivienda y el acceso a la salud. Cada uno de los sub-índices muestra de manera particular el grado de afectación o la magnitud del problema que se está midiendo, sin que alguno de ellos se relacione entre sí. Es decir, cada uno de los sub-índices sirven para alimentar al IPH, sin embargo, entre estos no existe ningún tipo de correlación, al menos a nivel estadístico; la pobreza es un problema cimentado en una serie de factores que se interrelacionan y potencian de manera conjunta.

\section{Los cálculos del índice}

Como se mencionó antes, el IPH se basa en cuatro variables que miden el ingreso (P1), la educación (P2) , los servicios de la vivienda (P3) y el acceso a salud (P4).

Al primer subíndice se le nombró P1 y muestra la variable ingreso. Sin embargo, debido a que los datos recopilados en el censo de 2010 no incluyen a la población que percibe entre 1 y 2 salarios mínimos, ${ }^{7}$ se utiliza a la población sin empleo como una aproximación de la misma.

- P1= Población sin empleo

Población económicamente activa

7 En estudios anteriores, la población que obtiene menos de 1 y 2 salarios mínimos de ingreso al día se caracteriza por ser la más vulnerable en términos económicos. 
Al segundo subíndice se le nombró P2 y se orienta a medir el nivel educativo de la población, por lo cual se utilizaron las variables siguientes:

- $\mathrm{P} 2$ = Población de 15 años y más con primaria incompleta Población total de 15 años y más

Al tercer subíndice se le nombró P3 y mide los servicios al interior de la vivienda, las variables que se utilizaron son:

- $\mathrm{P} 3=(1)$ - (Promedio de ocupantes de la vivienda* viviendas particulares que disponen de agua, drenaje y energía eléctrica) $)^{*} 100$

Población total

Finalmente, al subíndice que mide salud se le nombró P4.

- $\mathrm{P} 4=$ Promedio de hijos nacidos vivos.

\section{Los resultados del IPH}

La medición del IPH para el año 2010, arrojó un índice de 24.06 $\%$, lo que significó que para dicho año la pobreza humana fue superior en el municipio que la detectada en el país. ${ }^{8}$ En cuanto a los resultados particulares, prevalece el nivel medio de pobreza para toda la ciudad, aunque también fue posible detectar AGEB con niveles de pobreza alto. Respecto a los datos absolutos se obtuvo que trece mil personas experimentan algún grado de pobreza humana, sin embargo, hay que resaltar que los resultados obtenidos no son completamente coincidentes con los otorgados por el Coneval, ya que este situaba alrededor del $39.2 \%$ de la población del municipio en pobreza patrimonial. Aunque también es preciso decir que la metodología del Coneval se orienta a medir los ingresos de las personas y a partir de ello considerar quién es pobre y quién no, mientras que la metodología que empleamos nosotros se ubica en el rubro multidimensional, aspectos que de entrada son metodológicamente diferentes y que explican las di-

8 La primera medición realizada por el PNUD de la ONU, situó a México con un IPH de .09 
ferencias respecto a los resultados obtenidos (véanse cuadros 3 , 4 y 5 , y gráfico 1 ).

Cuadro 3: Estratos del IPH, Nuevo Casas Grandes, 2010

\begin{tabular}{cc}
\hline IPH & Valor \\
\hline Muy alto & 41 y más \\
\hline Alto & 31 hasta 40 \\
\hline Medio & 21 hasta 30 \\
\hline Bajo & 11 hasta 20 \\
\hline Muy bajo & 0 a 10
\end{tabular}

Fuente: elaborado por los autores, 2016

Cuadro 4: Población por estrato del IPH, Nuevo Casas Grandes, 2010

\begin{tabular}{lccccc}
\hline \multicolumn{1}{c}{ Estratos } & 0 a $\mathbf{1 0}$ & 11 a $\mathbf{2 0}$ & 21 a $\mathbf{3 0}$ & 31 a $\mathbf{4 0}$ & 41 y más \\
\hline AGEB & 8 & 10 & 26 & 6 & 0 \\
\hline Población & 15 & 14903 & 29794 & 10841 & 0 \\
\hline Pob. total & 55553 & & & & \\
\hline AGEB no incluidas & 4 & & & & \\
\hline Pob. no incluida & 0 & & & & \\
\hline
\end{tabular}

Fuente: elaborado por los autores, 2016

Cuadro 5: Resultados generales del IPH, Nuevo Casas Grandes, 2010

\begin{tabular}{ccccc}
\hline IPH & P1 & P2 & P3 & P4 \\
\hline 24.06 & 5.12 & 32.62 & 3.18 & 2.1 \\
\hline
\end{tabular}

Fuente: elaborado por los autores, 2016 
Gráfico 1. Índice de pobreza humana para el municipio de Nuevo Casas Grandes, 2010

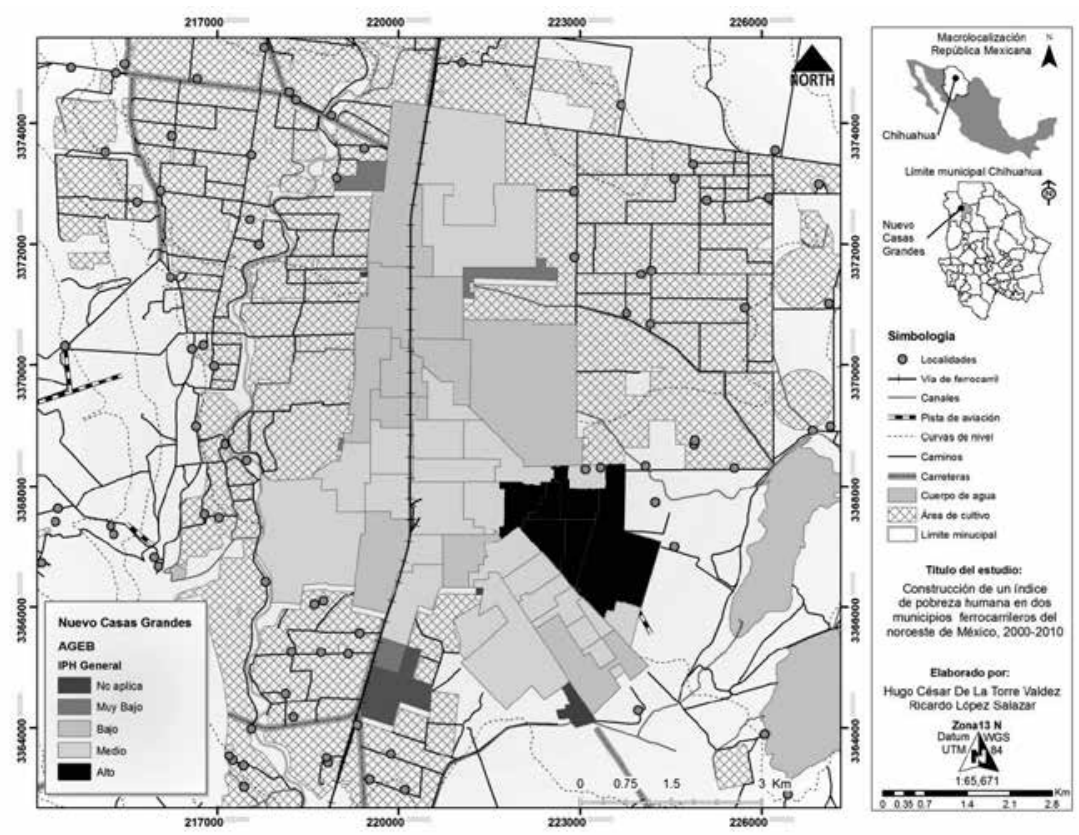

Fuente: elaborado por los autores, 2016

Ingreso-empleo, 2010

Debido a que los tabulados básicos del Censo de 2010 no incluyen a la población que obtiene uno y dos salarios mínimos al día de ingreso, los cuales se caracterizan por ser insuficientes para situarse por encima de la línea de bienestar mínimo, utilizamos a la población sin empleo para tener una aproximación adecuada. Sin embargo, es pertinente mencionar que la utilización de las personas desempleadas como parte de la medición involucra la posibilidad de obtener tres efectos, tanto para el subíndice como para el IPH.

En primer lugar, obtendríamos el promedio de la población sin ingreso producto de la falta de empleo, aunque en algunos estratos, buena parte de sus ingresos podrían no provenir de un trabajo sino de los programas sociales del gobierno federal, como Oportunidades y el PAL, así como de otras transferencias en es- 
pecie provenientes de familiares o amistades, por lo que se podría hacer una sobreestimación en el cálculo. En segundo lugar, al introducir a la población sin empleo dentro de la medición, se obtiene a las personas que en caso de no obtener un ingreso por otra vía prácticamente se situarían en el nivel más alto de pobreza. En tercer lugar, debido a que la tasa de desempleo en México y en general en todos los países latinoamericanos es relativamente baja debido a la alta informalidad del trabajo, ello sin duda provoca que al tomar como variable central al desempleo para medir el ingreso de la población, los cálculos resulten con menores valores a los que se obtendrían si se toma a la población que obtiene entre uno y dos salarios mínimos, lo cual repercute tanto en P1 como en el IPH.

Como se alertó en líneas anteriores, la prevalencia del desempleo es relativamente baja ${ }^{9}$ (probablemente por la alta informalidad del trabajo), aunque existen algunos AGEB que registran tasas medias de desempleo, y las hay en menor número con tasas muy altas. La tasa de desempleo más alta ronda el 14 \% y la más baja alrededor del $1.8 \%$, mientras que, en términos de espacio, los niveles de desempleo medio se ubican en las periferias de la ciudad (véase gráfico 2).

9 En 2010, la tasa de desempleo abierto en México fue del $5.3 \%$, es decir, casi en el mismo nivel que la exhibida por Nuevo Casas Grandes para dicho año. 
Gráfico 2: Población sin empleo en Nuevo Casas Grandes, 2010

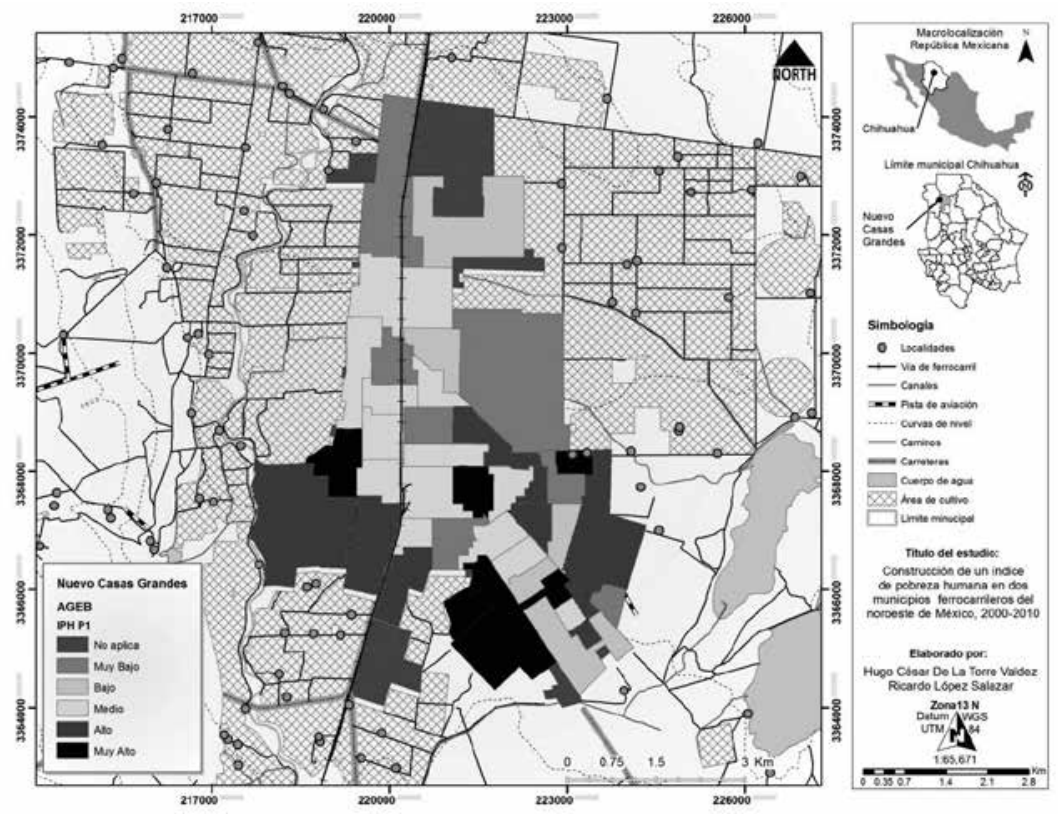

Fuente: elaborado por los autores, 2016

Acceso a la educación, 2010

Respecto a los niveles de educación de la población, prevalece una privación entre medio y alto. En términos absolutos, alrededor de 18000 personas mayores de quince años no cuentan con la primaria concluida. En cuanto a su localización geográfica, las áreas de nivel medio y alto, respecto a la privación del acceso a la educación, se encuentran dispersas por todo el municipio, ya que tanto la zona sur, la zona centro y la parte oeste del municipio alojan a una cantidad importante de personas con carencias educativas.

Resulta evidente el reto en el tema educativo, sobre todo si consideramos la alta presencia de analfabetas así como la altísima cifra de personas sin primaria concluida, los cuales al conjugarse derivan en aspectos que limitan sus posibilidades para integrarse a los mercados laborales, sobre todo a aquellos que demandan personal capacitado y adiestrado. De esta manera, aunque en Nuevo Casas Grandes se concentraban instituciones 
de educación media superior como Cobach, CBTA, Cetys y centros de nivel superior como la UACJ, UPN, URN, UTP, por citar algunos, ello fue insuficiente para darle un giro radical en cuanto al rezago educativo de la ciudad (véase gráfico 3).

Gráfico 3: Población de 15 años y más con primaria incompleta, Nuevo Casas Grandes, 2010



Fuente: elaborado por los autores, 2016

Servicios al interior de la vivienda, 2010

Por su parte, el indicador que hace referencia a los servicios con los cuales cuentan las viviendas del municipio, da cuenta de que existe un rezago relativamente pequeño en los mismos, ya que su valor alcanza casi el 3.18 por ciento. En otras palabras, si normalizamos el dato encontramos que alrededor de dos mil personas habitan en viviendas que no cuentan con todos los servicios, como luz eléctrica y agua potable (véase gráfico 4). En adición, y considerando que en promedio las familias del municipio están 
integradas por cinco habitantes, el número de viviendas con servicios inadecuados alcanza alrededor de $400 .{ }^{10}$

En relación con su distribución geográfica, es claro que en las partes periféricas de la ciudad se observa una presencia mayor de viviendas clasificadas con valores de medio a muy alto en cuanto a la falta de agua, drenaje o luz eléctrica.

Gráfico 4: Población con servicios básicos al interior de la vivienda, Nuevo Casas Grandes, 2010

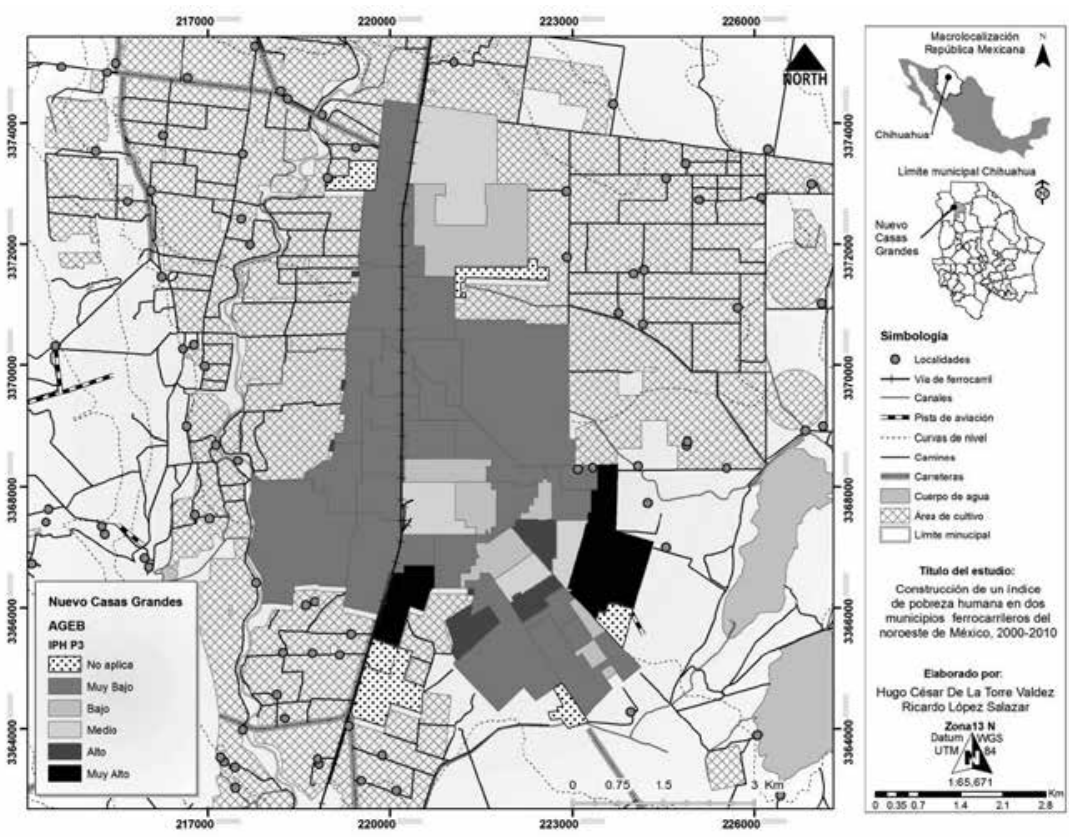

Fuente: elaborado por los autores, 2016

\section{El acceso a salud, 2010}

Uno de los ejes fundamentales para analizar la calidad de vida de las personas, sin duda, es el componente de salud y el acceso a la misma. De esta forma, el índice P4 está orientado a medir la tasa

10 Este dato resulta de dividir a las 2000 personas que habitan viviendas con servicios inadecuados entre el promedio de integrantes del hogar en la ciudad, que es de cinco. Por supuesto que dicho dato es ilustrativo y, por tanto, puede estar sobreestimando el problema. Sin embargo, por lo menos otorga una aproximación con base en cifras fiables. 
de mortalidad de los niños recién nacidos, el cual se considera un indicador primario para medir el avance o estancamiento en el acceso a la salud de las personas. Los resultados que obtuvimos sitúa al índice en 2.1, lo que resulta el valor más bajo de todos los cálculos que se realizaron. Ante dicho resultado es posible afirmar que la tasa de mortalidad de niños recién nacidos en la ciudad es sumamente baja, lo que es muestra incuestionable del avance en la materia.

El rango más alto de mortalidad infantil alcanzó un valor de 3.8, mientras que el más bajo fue de cero, es decir, existen algunos AGEB que tienen una tasa de mortalidad infantil más alta y otros prácticamente nula. La distribución geográfica conduce a mencionar la predominancia del nivel bajo a medio, mientras que solo cuatro AGEB resultan en el nivel de muy alto (véase gráfico 5).

Gráfico 5: Acceso a salud, Nuevo Casas Grandes, 2010

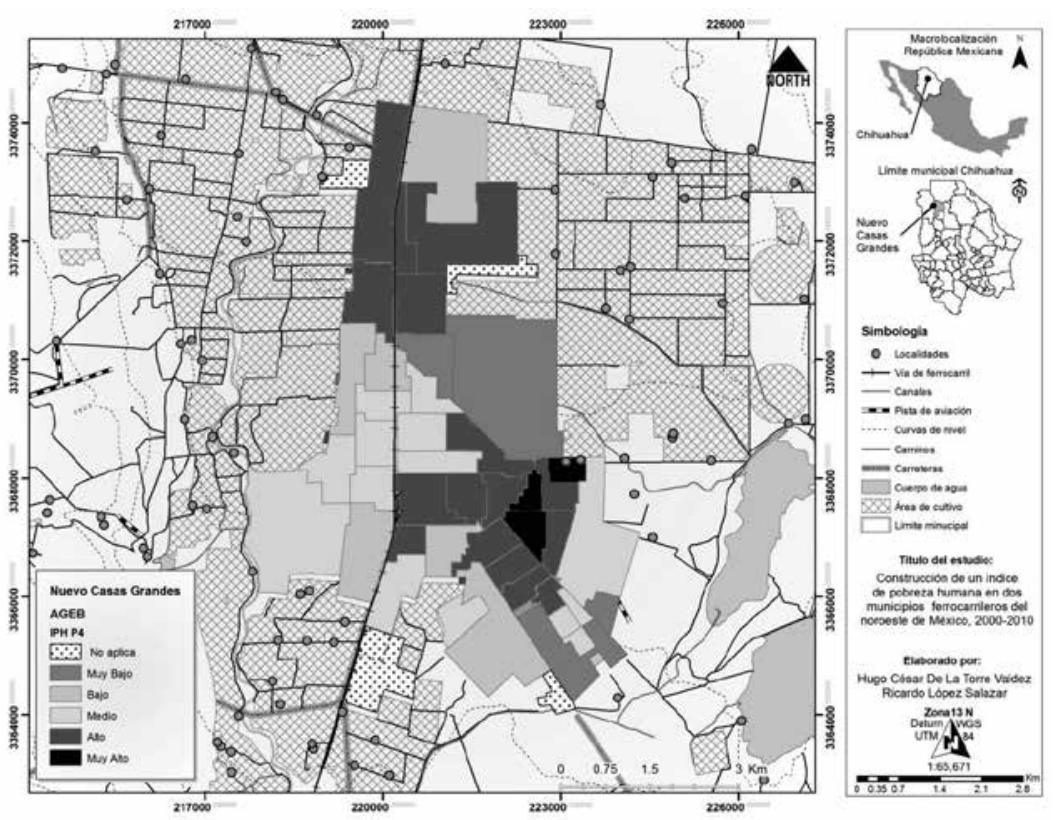

Fuente: elaborado por los autores, 2016 


\section{CONCLUSIONES}

Con el proceso de descentralización administrativa puesta en marcha en el país, los municipios han tomado un "nuevo aire" en cuanto a su intervención en los asuntos gubernamentales y de interés público. Es común ahora que el municipio elabore planes y políticas públicas que anteriormente no efectuaban debido a la carencia de recursos económicos y, principalmente, por la falta de una figura administrativa sólida, la cual se le concedió con las reformas de 1997.

Se puede afirmar que existen avances promisorios en el ámbito municipal, gracias al proceso de descentralización administrativa y al empuje de los propios gobiernos municipales. Sin embargo, este proceso aún se encuentra en ciernes y muestra un grado de avance que hasta el momento no es suficiente para situar al municipio al nivel de importancia que poseen los estados y la federación.

Por su parte, los planes de desarrollo municipal, sin duda alguna, exponen las formas, los mecanismos, los instrumentos, las estrategias y las líneas de acción trazadas por las administraciones municipales, elemento vital para juzgar y evaluar su desempeño. Para el propósito de este documento, el análisis de los PDM otorga una visión amplificada sobre la actuación del municipio en el combate a la pobreza.

En primer lugar, se evidencia la carencia de un diagnóstico adecuado y consistente que otorgue no solamente datos, sino todo el mosaico de interrelaciones que se conjugan para detonar y agudizar la pobreza. En otras palabras, los PDM consultados no profundizan en las causas o en los fenómenos que interactúan al interior del municipio y, por ende, se constituyen en aspectos claves para comprender y visualizar los orígenes y causas del problema.

Como resultado de la carencia en el diagnóstico, las políticas contra la pobreza enunciadas en los PDM se caracterizan por ubicarse en el rubro asistencial, es decir, consisten en la otorgación de despensas, apoyos económicos y desayunos escolares. Sin embargo, este tipo de apoyos asistenciales solamente se concentran en grupos considerados como "marginados o vulnerables" como los niños, las madres solteras o trabajadoras y los adultos mayores. De 
esta manera, se pueden obtener al menos dos lecturas de lo anterior. En primer lugar, las políticas asistenciales básicamente son una estrategia de "alivio" a la pobreza alimentaria en el sentido de que su función primordial es disminuir la incidencia del problema en el corto plazo, disminuyendo su efectividad a largo plazo. En segundo lugar, las políticas son meramente focalizadas, alejadas de las políticas de cobertura universal aplicadas en el pasado.

En relación con el Índice de Pobreza Humana, los resultados obtenidos de los cálculos elaborados muestran un valor de 24, el cual es superior al obtenido para el caso de México (.09) y notablemente mayor que para otras ciudades que han sido sometidas al mismo estudio, como Hermosillo, con 7.71. Desafortunadamente, en Nuevo Casas Grandes parece estarse gestando un fenómeno poco halagador que combina estancamiento económico, niveles bajos de escolaridad y escasas oportunidades laborales. Como resultado, la pobreza y la marginación persisten en buena parte del municipio y, por ende, su combate y erradicación se debe configurar como uno de los objetivos primordiales del gobierno local. Sin embargo, para que ello ocurra primeramente es necesario que se incorpore la agenda pública con un enfoque de derechos humanos universales. El reto es enorme y demanda la atención de todos. El futuro no puede esperar.

\section{BIBLIOGRAFÍA}

Arellano Gault, David (2000). "El municipio y las políticas sociales”, Agenda de la Reforma Municipal en México, Cuadernos de Debate, México, CIDE. Bardach, E. (1998). Los ocho pasos para el análisis de políticas públicas: un manual para la práctica. México, Centro de Investigación y Docencia Económica (CIDE).

Boltvinik, J. (2000). "Los métodos de medición de pobreza. Conceptos y tipología”, en Gallardo Gómez, Rigoberto y Osorio, Joaquín (coords.), Los rostros de la pobreza: el debate, México, Limusa/Iteso/Universidad Iberoamericana/sEUIA-tomo III.

Cabrero, Enrique (2005). Acción pública y desarrollo local, México, Fondo de Cultura Económica. 
Consejo Nacional de Evaluación de la Política Social (2009). Metodología para la medición multidimensional de la pobreza, México, Coneval.

Garza, Gustavo (2003). La urbanización de México en el siglo XX, México, El Colegio de México.

Gore, Charles (1984). Regions in questions space, development theory and regional policy, London, Methen \& Co. Ltd.

Gutiérrez, Manuel y Sarah Martínez (1994). "El papel de los gobiernos locales en el desarrollo regional”. Revista Gestión y Política Pública, vol. III, núm. 1.

Instituto Nacional de Geografía y Estadística (2010). Censo General de Población y Vivienda.

Martínez, Alejandra (2015). Análisis de las estrategias de marketing de las políticas públicas y planes de gobierno, referentes a las privatizaciones y combate a la pobreza, en dos municipios ferrocarrileros del noroeste de México: Empalme, Sonora y Nuevo Casas Grandes, Chihuahua. Tesis para obtener el grado de licenciatura en Mercadotecnia, UAcJ. Director: Ricardo López Salazar.

Merino, Mauricio (2006). La gestión profesional de los municipios en México: Diagnóstico, oportunidades y desafíos, México, CIDE-Sedesol, Banco Mundial.

Plan de Desarrollo Municipal, Nuevo Casas Grandes (2004-2007). Presidente Orlando Polanco Rascón.

Plan de Desarrollo Municipal, Nuevo Casas Grandes (2007-2010). Presidente Jesús Manuel Pendones.

Plan de Desarrollo Municipal, Nuevo Casas Grandes (2010-2013). Presidente Luis Fernando Cobos.

Plan de Desarrollo Municipal, Nuevo Casas Grandes (2013-2016). Presidente Rodolfo Soltero Aguirre.

Salama, Pierre (2011). Luchas contra la pobreza en América Latina: el caso de la pobreza rural en Brasil. Revista Problemas del Desarrollo, volumen 42, no. 165, UNAM.

Sen, Amartya (2000). Desarrollo y libertad. Barcelona, España, Editorial Planeta.

Spicker, Paul (1999). Definiciones de pobreza: doce grupos de significados, Clacso.

United Nations Development Programme (1997). Human Development Report, Oxford University Press. 